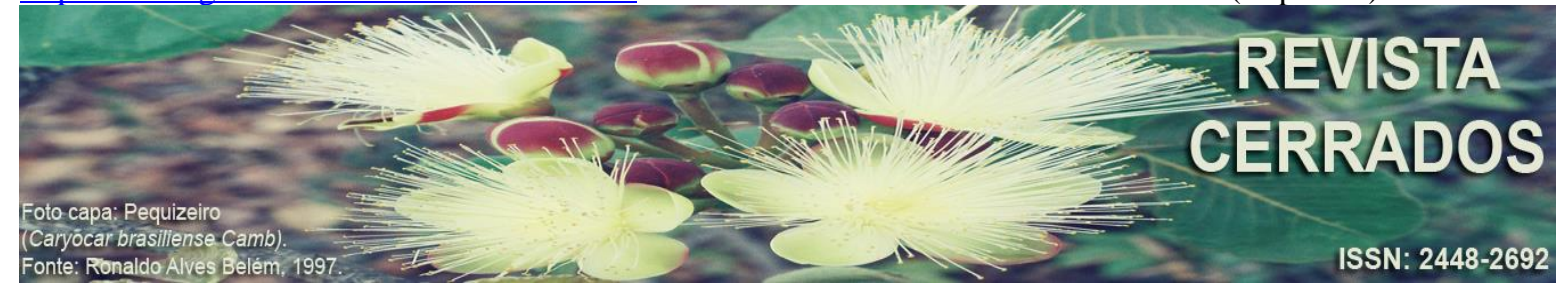

\title{
SENSORIAMENTO REMOTO NA DETECÇÃO E ANÁLISE DE FOCOS DE CALOR NA BACIA HIDROGRÁFICA DO RIO MUNIM
}

\section{REMOTE SENSING IN THE DETECTION AND ANALYSIS OF HEAT FOCUSES IN THE HYDROGRAPHIC BASIN OF RIO MUNIM}

\section{SENSOR REMOTO EN LA DETECCIÓN Y ANÁLISIS DE ENFOQUES DE CALOR EN LA CUENCA HIDROGRÁFICA DE RÍO MUNIM}

\author{
Rennato Oliveira da Silva \\ Universidade Federal do Maranhão - UFMA, Chapadinha, Maranhão, Brasil. \\ E-mail: <oliveira.rennato@hotmail.com>. \\ Rhuan Oliveira da Silva \\ Universidade Federal do Maranhão - UFMA, Chapadinha, Maranhão, Brasil. \\ E-mail: <rhuanoliveyra@gmail.com>. \\ Thais de Carvalho Araújo \\ Universidade Federal do Maranhão - UFMA, Chapadinha, Maranhão, Brasil. \\ E-mail: <araujothays01@gmail.com>. \\ Carlos Augusto Alves Cardoso Silva \\ Universidade Federal do Maranhão - UFMA, Chapadinha, Maranhão, Brasil. \\ E-mail: <carlosaugusto8435@gmail.com>. \\ Ana Karla da Silva Oliveira \\ Universidade Federal do Maranhão - UFMA, Chapadinha, Maranhão, Brasil. \\ E-mail: <karlinha_oliveira95@hotmail.com>. \\ Kamilla Andrade de Oliveira \\ Universidade Federal do Maranhão - UFMA, Chapadinha, Maranhão, Brasil. \\ E-mail: <kamilla.andrade@ufma.br>.
}

\section{RESUMO}

Os incêndios são a causa da devastação de milhares de hectares de ecossistemas do planeta, gerando impactos à saúde pública, prejuízos econômicos e ambientais. O presente estudo tem como objetivo realizar uma análise temporal dos focos de calor detectados em diferentes usos 
SIlVA, R. O.; ARAÚJO, T. C.; SIlVA, R. O.; SIlvA, C. A. A. C.; OLIVEIRA, A. K. S.; OLIVEIRA, K. A. Sensoriamento remoto na detecção e análise de focos de calor na bacia hidrográfica do Rio Munim

e cobertura da terra mapeados entre 2008 e 2018 na Bacia Hidrográfica do Rio Munim (BHRM), leste Maranhense. Os dados utilizados no trabalho foram obtidos junto à plataforma digital do Instituto Nacional de Pesquisas Espaciais (INPE), sendo provenientes dos satélites TERRA, AQUA, GOES, NOAA, MSG-02 e ERS-2. Constatou-se um total de 74.752 ocorrências de focos de incêndios na área da bacia entre os anos de 2008 a 2018, tendo como destaque o mês de novembro o mais crítico registrando $30,33 \%$ do total de focos, seguido pelo mês de outubro com $23,03 \%$, período em que praticamente não existe precipitação na região. Verificou-se que o ano de 2015 foi o que apresentou o maior índice de focos com 14.025 ocorrências e que o ano de 2011 foi o menos expressivo, com 2.116 ocorrências.

Palavras-chave: Geotecnologias. Uso do solo. Monitoramento.

\begin{abstract}
Fires are the cause of the devastation of thousands of hectares of ecosystems on the planet, generating impacts on public health, economic and environmental losses. The present study aims to perform a temporal analysis of the heat sources detected in different uses and land cover mapped between 2008 and 2018 in the hydrographic basin of the Munim River, eastern Maranhense. The data used in the study were obtained from the digital platform of the National Institute for Space Research (INPE). The number of outbreaks recorded came from the TERRA, AQUA, GOES, NOAA, MSG-02 and ERS-2 satellites. A total of 74,752 outbreaks in the basin area were found between the years 2008 to 2018, with November as the most critical month, with $30.33 \%$ of total outbreaks, followed by October with $23.03 \%$, a period in which there is practically no rainfall in the region. It was found that the year 2015 was the one with the highest rate of outbreaks with 14,025 occurrences and that the year 2011it was the least expressive, with 2,116 occurrences.
\end{abstract}

Keywords: Geotechnologies. Use of the soil. Monitoring.

\title{
RESUMEN
}

Los incendios son la causa de la devastación de miles de hectáreas de ecosistemas en el planeta, generando impactos en la salud pública, pérdidas económicas y ambientales. El presente estudio tiene como objetivo realizar un análisis temporal de las fuentes de calor detectadas en diferentes usos y la cobertura del suelo mapeada entre 2008 y 2018 en la cuenca hidrográfica del río Munim, este de Maranhense. Los datos utilizados en el estudio se obtuvieron de la plataforma digital del Instituto Nacional de Investigación Espacial (INPE). El número de brotes registrados provino de los satélites TERRA, AQUA, GOES, NOAA, MSG02 y ERS-2. Se encontraron un total de 74,752 brotes en el área de la cuenca entre los años 2008 hasta 2018, con noviembre como el mes más crítico, con 30.33\% de los brotes totales, seguido de octubre con $23.03 \%$, un período en el que prácticamente no hay precipitaciones en la región. Se encontró que el año 2015 fue el que tuvo la mayor tasa de brotes con 14.025 ocurrencias y que el año 2011 fue el menos expresivo, con 2,116 ocurrencias.

Palabras-clave: Geotecnologías. Uso del suelo. Monitoreo. 
SILVA, R. O.; ARAÚJO, T. C.; SILVA, R. O.; SILVA, C. A. A. C.; OLIVEIRA, A. K. S.; OLIVEIRA, K. A. Sensoriamento remoto na detecção e análise de focos de calor na bacia hidrográfica do Rio Munim

\section{INTRODUÇÃO}

O fogo é um agente físico importante que pode levar a alterações nas propriedades físicas, químicas e biológicas do solo (THOMAZ et al., 2014). Os incêndios florestais destroem milhares de hectares dos ecossistemas em diversos biomas ocasionando diversos impactos: ambientais, na saúde pública intensificando os prejuízos econômicos e socioambientais (JUSTINO, 2002; IMESC, 2015). No Brasil, as queimadas são técnicas utilizadas na agricultura, tanto na sua forma primitiva, como em sistemas de produção intensificados, atuando no sentido de eliminarem resíduos, pragas ou ainda de renovação de pastos (MIRANDA, 2002).

A expansão da fronteira agrícola, a extração madeireira, o desmatamento, e as mudanças climáticas aliadas aos eventos de extremos climáticos intensos das últimas décadas, tem afetado as florestas e sua resistência natural ao fogo (IPCC, 2018). Principalmente em decorrência de períodos maiores de estiagem e maior número de focos de calor. Os processos antrópicos, no uso do fogo e práticas que o favoreçam, juntamente com os efeitos gerados pelas mudanças climáticas têm afetado as condições ambientais e proporcionalmente o uso da terra, influenciado pelas fontes e o aumento da ocorrência de fogo (NEPSTAD et al., 2008).

A identificação das alterações no uso e cobertura do solo, ocorridas ao longo do tempo, devido a atividades antrópicas são de grande importância para um melhor planejamento e gestão dos recursos naturais (SILVA et al., 2018). Uma vez que, o termo o uso e a cobertura do solo não partilham do mesmo sentido, dada a etimologia das palavras, frequentemente utilizados como tendo a mesma definição (DIMYATI et al., 1996).

Neste contexto, a vegetação considerada um indicador de qualidade ambiental, na medida em que atua associada a outros indicadores, tais como qualidade da água, do ar, solos, fauna e clima, apresenta-se como elemento indispensável ao equilíbrio, seja na manutenção e serviços ecossistêmicos, seja nas ações que visam a melhoria da qualidade de vida em áreas mais comprometidas (SOUSA, 2008).

Na região do semiárido brasileiro, é prática cultural das populações realizarem a remoção de vegetação, visando o uso alternativo do solo para atividades agropecuárias e extração vegetal. Além disso, a produção agropecuária, se praticada de forma incorreta, pode causar danos ao meio ambiente, contribuindo para a degradação dos meios físico e biótico (OLIVEIRA; OLIVEIRA, 2017). Segundo Araújo et al. (2013), as queimadas e incêndios 
SILVA, R. O.; ARAÚJO, T. C.; SILVA, R. O.; SILVA, C. A. A. C.; OLIVEIRA, A. K. S.; OLIVEIRA, K. A. Sensoriamento remoto na detecção e análise de focos de calor na bacia hidrográfica do Rio Munim

florestais contribuem na emissão de $\mathrm{CO}^{2}$, levando a uma retroalimentação ao sistema climático, sendo que, a emissão deste gás intensifica o efeito estufa e propicia condições meteorológicas ainda mais favoráveis às ocorrências de casos de ineficiência das técnicas de controle utilizados em queimadas prescritas e aos incêndios florestais.

A ação do fogo infelizmente ainda é bastante utilizado no Brasil para fins de manejo de pastagem e limpeza de áreas para a agricultura tradicional, pelo fato de ser um método mais fácil, rápido e barato, além disso, é a forma como muitos produtores estimula a rebrota da pastagem, diminui as pragas e remove os remanescentes agrícolas (ALVES; HOMMA, 2020; SILVA et al., 2018; IMESC, 2017).

O uso do fogo como uma técnica de preparo dos terrenos visando a agricultura é atribuído equivocadamente aos povos tribais de floresta (LEONEL, 2000). Dentre as atividades antropogênicas, as queimadas são as principais responsáveis pela liberação de partículas de aerossóis para o ar e essas partículas interagem com a radiação solar afetando o balanço de energia, bem como alterando o ciclo hidrológico local (NOVAIS et al., 2017).

Os incêndios florestais provocam alterações significativas em vários componentes do ciclo hidrológico, nomeadamente ao nível da intercepção pela copa das árvores, na evapotranspiração para a atmosfera, na capacidade de infiltração dos solos, e nos processos pelos quais a água chega aos cursos de água e aos aquíferos, influenciando assim o cálculo de base e os picos de cheia das bacias hidrográficas (MARTIN e MOODY, 2001; MEYER, 2002).

De acordo com estudos (MARTIN e MOODY, 2001; MEYER, 2002; NEARY et al., 1999) quando o incêndio consome a vegetação e a manta morta subjacente, o solo fica exposto e a sua porosidade pode diminuir devido ao impacto direto das gotas de chuva resultando em quantidades de escorrência muito maiores. Para além de aumentar drasticamente a energia do impacto das gotas de chuva, a perda de vegetação e da manta morta na sequência de um incêndio reduz ainda mais a capacidade de retenção e armazenamento da água bem como a resistência aos fluxos de água nas vertentes, também em resultado da perda da matéria orgânica do solo.

Existem diversas formas de detectar incêndios, como vigilância terrestre, posto de observação ou patrulhamento aéreo e/ou monitoramento por imagens de satélites (SOARES; BATISTA; TETTO, 2017). Portanto, para países com grande extensão territorial como o Brasil, o uso das técnicas de sensoriamento remoto, que são mais rápidas e com menor custo, 
SILVA, R. O.; ARAÚJO, T. C.; SILVA, R. O.; SILVA, C. A. A. C.; OLIVEIRA, A. K. S.; OLIVEIRA, K. A. Sensoriamento remoto na detecção e análise de focos de calor na bacia hidrográfica do Rio Munim

garantindo eficiência para monitoramento e gerenciamento de diversas regiões remotas, os quais podem gerar ações para fiscalizar e minimizar os incêndios florestais (Pereira e Silva, 2016; BATISTA, 2004).

Neste sentido, o uso das geotecnologias para a análise da distribuição espacial de dados de incêndios florestais se torna fundamental, pois alia técnicas que permitem gerenciamento e monitoramento territorial (GOMES, 2006; VETTORAZZI; FERRAZ, 1998). Pois, os produtos originados de técnicas de geoprocessamento viabilizam o estabelecimento de ações de políticas públicas de controle e combate aos incêndios permitindo localizar, quantificar e fazer análises espaço-temporais. Nesse sentido, o presente estudo teve como objetivo realizar uma análise temporal dos focos de calor associados com a dinâmica do uso do solo na BHRM no período de 2008 e 2018.

\section{MATERIAL E MÉTODOS}

\section{Área de estudo}

A BHRM (figura 1) está localizada na região extremo leste do Estado do Maranhão, possui uma área total equivalente a 15.918,28 $\mathrm{km}^{2}$ correspondendo a 4,79\% do território do estado. O curso principal do rio possui $371,74 \mathrm{~km}$ de extensão e deságua na baía de São José, entre os municípios de Axixá e Icatu. Suas nascentes estão localizadas nos Tabuleiros de Formação Barreiras, a Nordeste da cidade de Caxias, tendo como principais afluentes a margem esquerda os rios Iguará, Paulica, riacho Mocambo, Raiz, da Cruz e São Gonçalo pela margem direita, os rios Preto, riacho Pirangi, Una e da Mata (MARANHÃO, 2016).

Dados do IBGE (2010) indicam que a área da BHRM é composta por 27 municípios, destes, 15 com sedes dentro da bacia. Com uma população de 320.001 habitantes, na BHRM, aproximadamente 4,90\% da população do Estado, destes 164.905 (51,50\%) estão situados na zona urbana e 155.093 (48,50\%) na zona rural, com uma densidade demográfica de 20,20 hab. $/ \mathrm{km}^{2}$. 
SIlVA, R. O.; ARAÚJO, T. C.; SIlVA, R. O.; SIlVA, C. A. A. C.; OLIVEIRA, A. K. S.; OLIVEIRA, K. A. Sensoriamento remoto na detecção e análise de focos de calor na bacia hidrográfica do Rio Munim

Figura 1. Mapa de localização da bacia hidrográfica do Rio Munim - MA

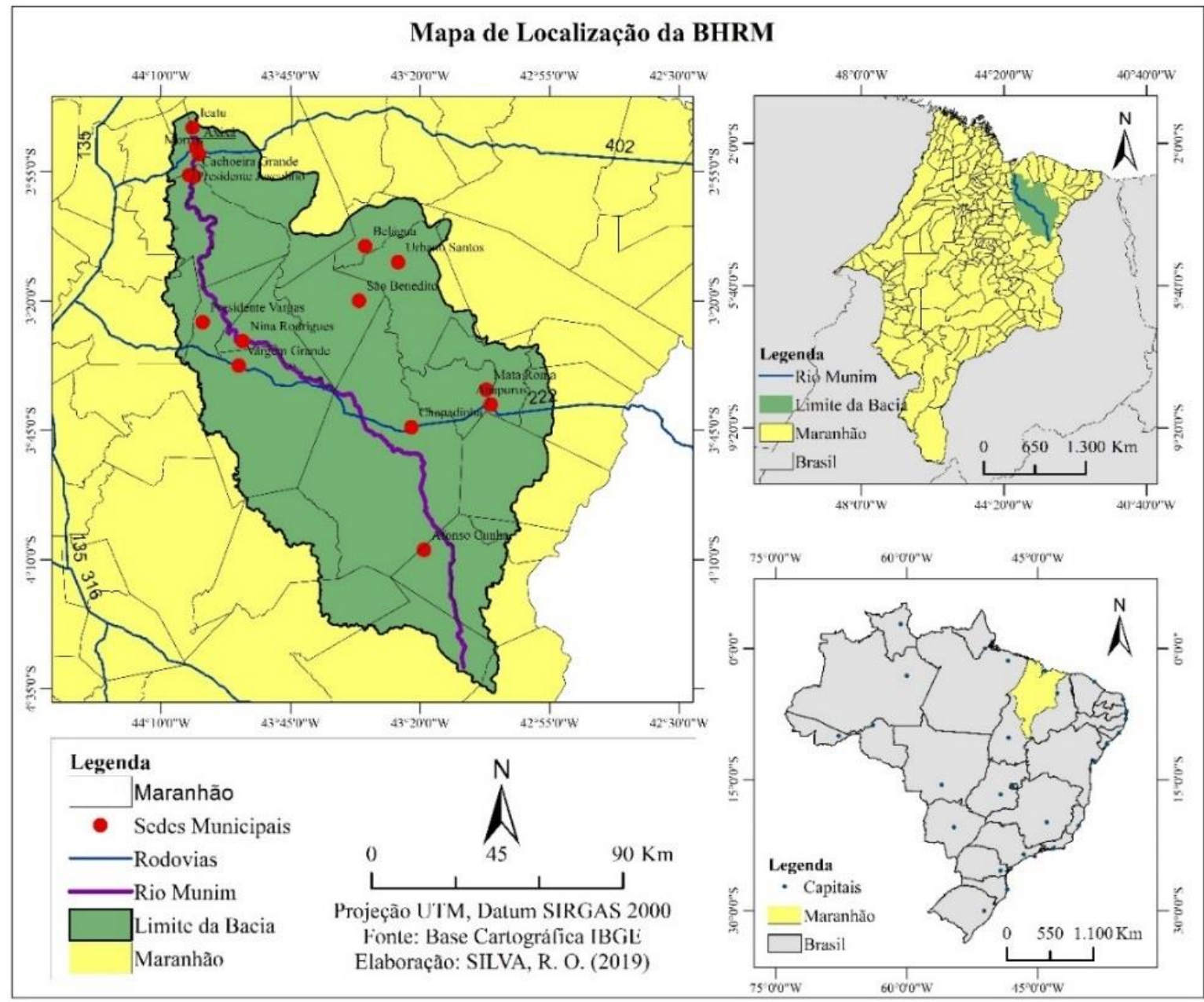

Fonte: Autores (2019).

Segundo Maranhão (2016), os dados climatológicos da BHRM correspondem principalmente ao clima úmido e sub-úmido, com acumulado anual de precipitação pluviométrica que variam de $1400 \mathrm{~mm}$ a $2400 \mathrm{~mm}$, sendo que as maiores precipitações são encontradas em áreas da região norte da bacia, valores significativos que refletem a grande influência da Zona de Convergência Intertropical nessa região. Por outro lado, os menores valores de precipitação pluviométrica são encontrados na região sul da bacia.

A umidade relativa do ar fica em torno de $70 \%$ a $82 \%$ com temperatura média anual registrada próximo de $25,6{ }^{\circ} \mathrm{C}$ sendo que os menores valores de temperaturas são registrados na região norte da bacia aproximadamente nas mesmas áreas que detém os maiores índices pluviométricos, visto que as chuvas podem amenizar os valores de temperatura do ar (MARANHÃO, 2016). 
SILVA, R. O.; ARAÚJO, T. C.; SILVA, R. O.; SILVA, C. A. A. C.; OLIVEIRA, A. K. S.; OLIVEIRA, K. A. Sensoriamento remoto na detecção e análise de focos de calor na bacia hidrográfica do Rio Munim

\section{Dados utilizados}

Os dados utilizados no trabalho foram obtidos junto ao "Banco de Dados Queimadas" disponível na plataforma digital do Instituto Nacional de Pesquisas Espaciais (INPE), em formato shapefile. Os dados são provenientes dos satélites TERRA, AQUA, GOES, NOAA, MSG-02 e ERS-2, cujos dados diários de focos de queimada registrados compõem uma série temporal ao longo dos anos permitindo a análise de tendências nos números de focos para mesmas regiões (INPE, 2007). Todos os satélites utilizados são considerados satélites de referência pelo INPE para o período em estudo. Utilizou-se diferentes sensores a fim de se obter a maior quantidade e diversidade de focos de queimadas registradas. Vale ressaltar que nem sempre a detecção remota dos focos de queimada indica a existência de fogo, apesar de a maioria estar relacionada diretamente a áreas com presença de fogo (BARBOSA, 2010).

Os dados obtidos, que correspondem ao intervalo de anos entre 2008 e 2018, foram organizados em uma única base de dados, para facilitar a manipulação e a análise da distribuição temporal (anos e meses) dos focos de queimada. Todo o processamento de dados foi realizado no software ArcMap versão 10.6, além disso, o software Microsoft Excel versão 2019 foi utilizado para gerar mapas de ocorrência anual e mensal de focos de calor na BHRM.

\section{RESULTADOS E DISCUSSÃO}

Após o tratamento e interpretação dos dados constatou-se um total de 74.752 ocorrências de focos de calor entre janeiro de 2008 a dezembro de 2018 nos municípios que estão inseridos na bacia hidrográfica do rio Munim. Na Tabela 1 verifica-se que o ano de 2015 foi o que apresentou o maior índice de focos, com 14.025 ocorrências, seguido dos anos de 2012, 2016 e 2017 com 11.731, 10.019 e 9.380 focos, respectivamente. O intervalo de anos entre 2008 a 2011 foram os menos significativos em relação à intensidade de focos, registrando apenas 11.498 ou 15,38\%. Destes, o ano de 2011 foi o menos expressivo, com apenas $2,84 \%$ do total. 
SILVA, R. O.; ARAÚJO, T. C.; SILVA, R. O.; SILVA, C. A. A. C.; OlIVEIRA, A. K. S.; OLIVEIRA, K. A. Sensoriamento remoto na detecção e análise de focos de calor na bacia hidrográfica do Rio Munim

Tabela 1. Focos de calor detectados entre 2008 e 2018 na Bacia Hidrográfica do Rio Munim

\begin{tabular}{lccccccccccc}
\hline Mês/ano & $\mathbf{2 0 0 8}$ & $\mathbf{2 0 0 9}$ & $\mathbf{2 0 1 0}$ & $\mathbf{2 0 1 1}$ & $\mathbf{2 0 1 2}$ & $\mathbf{2 0 1 3}$ & $\mathbf{2 0 1 4}$ & $\mathbf{2 0 1 5}$ & $\mathbf{2 0 1 6}$ & $\mathbf{2 0 1 7}$ & $\mathbf{2 0 1 8}$ \\
\hline Janeiro & 17 & 38 & 44 & 44 & 56 & 118 & 100 & 172 & 107 & 81 & 116 \\
Fevereiro & 7 & 4 & 30 & 8 & 32 & 20 & 19 & 30 & 40 & 20 & 5 \\
Março & 3 & 4 & 22 & 7 & 20 & 27 & 9 & 5 & 10 & 4 & 20 \\
Abril & 1 & 2 & 9 & 11 & 9 & 3 & 0 & 13 & 10 & 8 & 5 \\
Maio & 5 & 1 & 13 & 3 & 115 & 21 & 2 & 88 & 33 & 32 & 60 \\
Junho & 15 & 12 & 31 & 17 & 185 & 97 & 34 & 447 & 212 & 231 & 280 \\
Julho & 188 & 68 & 109 & 86 & 627 & 306 & 266 & 456 & 431 & 628 & 302 \\
Agosto & 303 & 270 & 272 & 228 & 1963 & 537 & 572 & 886 & 616 & 614 & 486 \\
Setembro & 230 & 221 & 415 & 374 & 2287 & 705 & 619 & 1609 & 843 & 1295 & 733 \\
Outubro & 647 & 268 & 528 & 206 & 3282 & 1605 & 1172 & 3620 & 2698 & 1895 & 1288 \\
Novembro & 1601 & 1252 & 1221 & 802 & 2164 & 1506 & 1927 & 3299 & 3564 & 2783 & 1436 \\
Dezembro & 481 & 551 & 449 & 340 & 991 & 1898 & 1052 & 3400 & 1455 & 1789 & 753 \\
\hline \multicolumn{1}{c}{ Total } & $\mathbf{3 4 9 8}$ & $\mathbf{2 6 9 1}$ & $\mathbf{3 1 9 3}$ & $\mathbf{2 1 1 6}$ & $\mathbf{1 1 7 3 1}$ & $\mathbf{6 8 4 3}$ & $\mathbf{5 7 7 2}$ & $\mathbf{1 4 0 2 5}$ & $\mathbf{1 0 0 1 9}$ & $\mathbf{9 3 8 0}$ & $\mathbf{5 4 8 4}$ \\
\hline
\end{tabular}

Fonte: Base de dados do INPE - Portal do Monitoramento de Queimadas e Incêndios, editado pelos autores (2019).

Diversos fatores podem influenciar o aumento ou a diminuição na intensidade de focos numa determinada região, dentre estes destacam-se o manejo e o uso do solo. Além de efeitos climatológicos/meteorológicos tais como El Niña e La Niña, tendo em vista que podem aumentar a quantidade de focos de calor devido ao aumento da seca e perda de umidade (SANTOS, 2011; LOPES et al, 2017).

Abreu e Souza (2016), estudando sobre a dinâmica espaço-temporal de focos de calor em duas terras indígenas no estado do Mato Grosso evidenciaram um aumento na intensidade de focos a partir do mês de julho com ápice em setembro, mês que correspondeu a 42,766\% (1526 focos). Relatam ainda, que esse período crítico (julho a agosto) está relacionado à baixa umidade do ar, pela ausência de precipitação e elevadas temperaturas, características atribuídas ao cerrado brasileiro. Nesse sentido, a figura 2 mostra a concentração mensal dos focos de calor e precipitação $(\mathrm{mm})$ observados para os anos de 2008 a 2018.

Analisando a figura 2, percebe-se que dentre os anos estudados o mês de novembro foi aquele em que mais se observou focos de calor, cerca de 30,33\% do total seguido pelo mês de outubro, com $23,03 \%$, período em que praticamente não existe precipitação. Esses resultados corroboram com os encontrados por Silva et al. (2013), quando estudavam a distribuição espacial dos focos de calor no Parque Nacional da Chapada Diamantina no Estado da Bahia entre os anos de 2000 a 2011, onde $89 \%$ do focos foram identificados nos meses de outubro e novembro em razão do aumento da temperatura e diminuição da umidade, favorecendo a combustão dos materiais depositados no solo.

Revista Cerrados, Montes Claros/MG, v. 18, n. 1, p. 373-388, jan./jun.-2020. 
SILVA, R. O.; ARAÚJO, T. C.; SILVA, R. O.; SILVA, C. A. A. C.; OLIVEIRA, A. K. S.; OLIVEIRA, K. A. Sensoriamento remoto na detecção e análise de focos de calor na bacia hidrográfica do Rio Munim

Figura 2. Distribuição mensal dos focos de calor e precipitação $(\mathrm{mm})$ para os anos de 2008 a 2018, sendo: (A) 2008; (B) 2009; (C) 2010; (D) 2011; (E): 2012; e (F) 2013; (G) 2014; (H) 2015; (I) 2016; (J) 2017 e (K) 2018
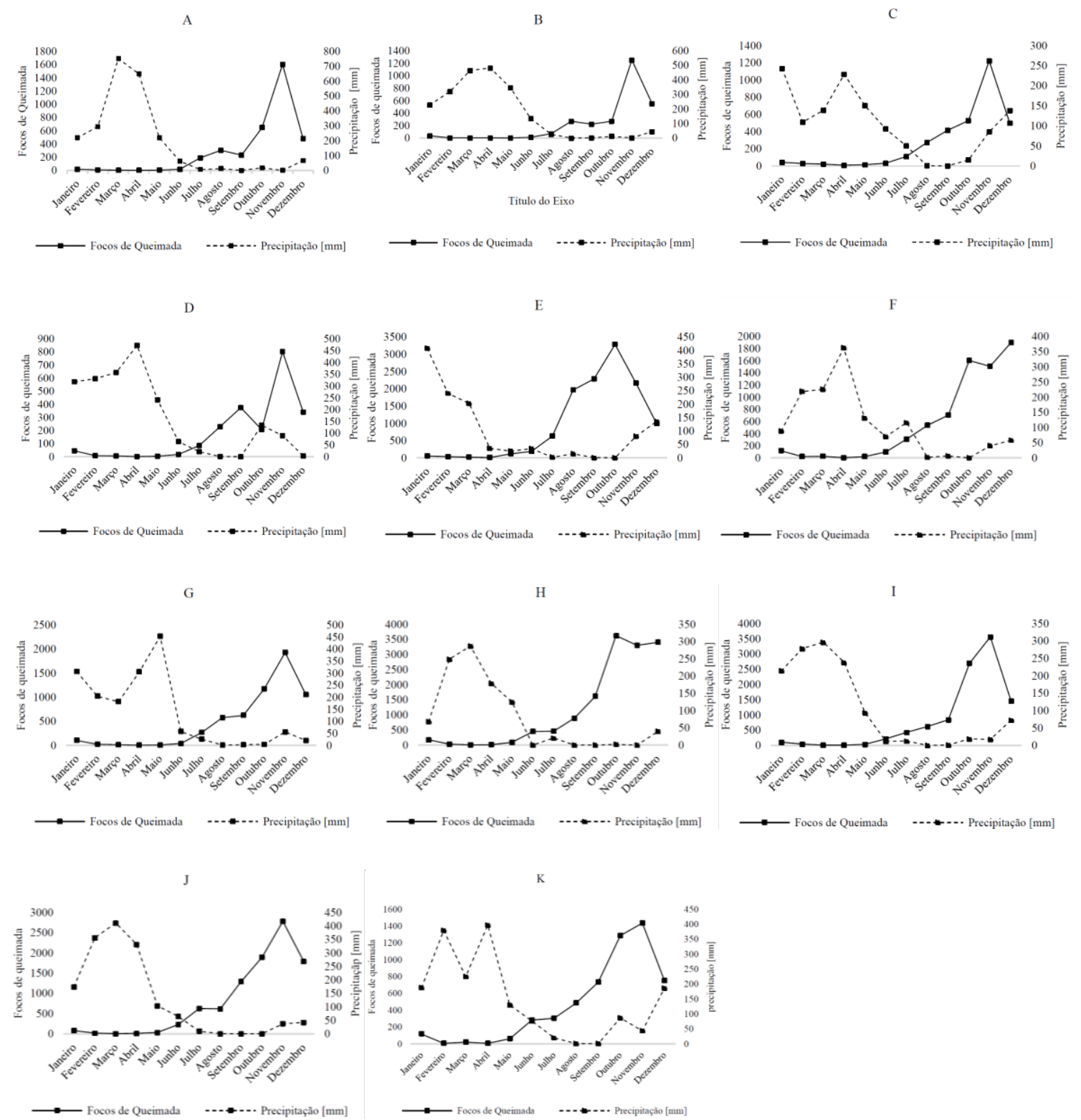

Fonte: Base de dados do INPE - Portal do Monitoramento de Queimadas e Incêndios, editado pelos autores (2019).

Lopes et al., (2017), analisando focos de calor no município de Novo Progresso no estado do Pará entre 2010 a 2015, observaram um aumento entre os meses de julho a novembro, principalmente nos meses de agosto e setembro, uma vez que somadas apresentam percentual de $89,73 \%$. Observaram ainda que existe uma relação direta entre a intensidade de focos com eventos meteorológicos, tendo em vista que nesse período do ano são menores a 
SILVA, R. O.; ARAÚJO, T. C.; SILVA, R. O.; SILVA, C. A. A. C.; OLIVEIRA, A. K. S.; OLIVEIRA, K. A. Sensoriamento remoto na detecção e análise de focos de calor na bacia hidrográfica do Rio Munim

ocorrência de precipitação, maiores temperaturas e menores taxas de umidade relativa do ar, favorecendo os incêndios originados pelas ações antrópicas. A figura 3 apresenta os mapas de densidade Kernel resultantes da interpolação dos focos de calor para cada ano em estudo, estimando-se a densidade por meio de 5 classes: muito baixa, baixa, média, alta e muito alta.

Figura 3. Mapas de densidade Kernel dos focos de calor para os anos de 2008 a 2018 na Bacia Hidrográfica do Rio Munim

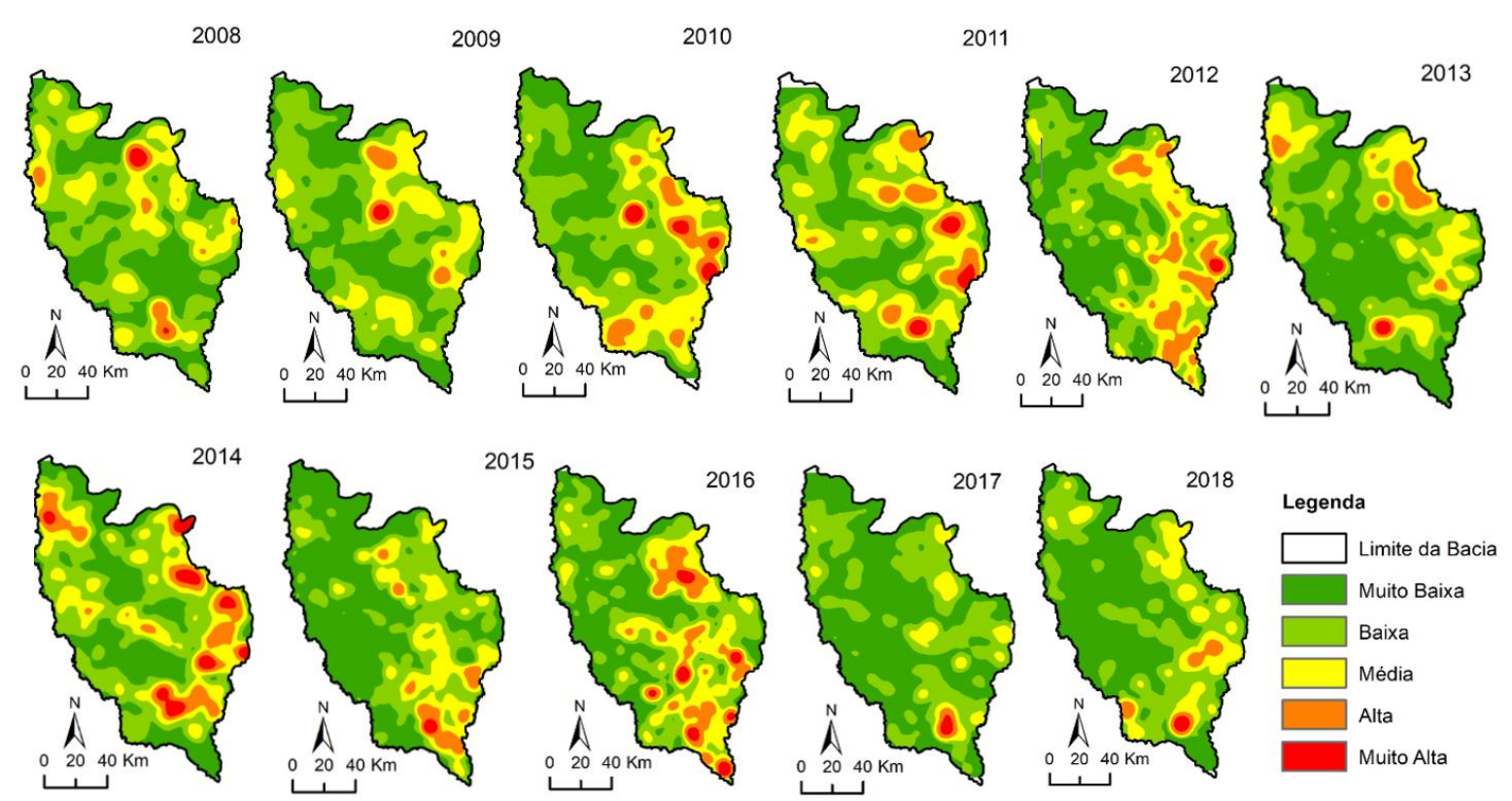

Fonte: Base de dados do INPE - Portal do Monitoramento de Queimadas e Incêndios, editado pelos autores (2019).

Segundo os dados obtidos dos mapas de densidade Kernel, nota-se que de maneira geral em todos os anos estudados as regiões Nordeste e Sul da bacia foram as mais afetadas pelas densidades alta e muito alta de focos, e isso se deve por essas regiões abrigarem sedes municipais ao longo da BR 222 e, principalmente pelo grande crescimento da monocultura soja na região, em áreas próximas à estrada pavimentada, os quais, tendem a ser valorizadas por diminuírem o custo de transporte e de produção.

A tabela 2 foi gerada a partir da quantificação das áreas classificadas nos mapas de densidade kernel sendo expressas em percentagens (\%). Nota-se que em todos os anos analisados as classes definidas como baixa, muito baixa apresentam percentuais compreendidos entre $24 \%$ a $48 \%$ e que a área de concentração definida como "muito alta" 
SIlVA, R. O.; ARAÚJO, T. C.; SIlVA, R. O.; SIlvA, C. A. A. C.; OLIVEIRA, A. K. S.; OLIVEIRA, K. A. Sensoriamento remoto na detecção e análise de focos de calor na bacia hidrográfica do Rio Munim

corresponde ao valor máximo de 3,33\% da área total da Bacia Hidrográfica do Rio Munin (BHRM), fato observado no ano de 2014.

Tabela 2. Percentual de cada região na BHRM correspondente s classe de concentração de focos de calor

\begin{tabular}{cccccc}
\hline Ano/Conc. & Muito alta & Alta & Média & Baixa & Muito baixa \\
\hline $\mathbf{2 0 0 8}$ & $0,75 \%$ & $3,56 \%$ & $17,37 \%$ & $48,63 \%$ & $29,70 \%$ \\
$\mathbf{2 0 0 9}$ & $0,49 \%$ & $3,06 \%$ & $20,30 \%$ & $43,26 \%$ & $32,89 \%$ \\
$\mathbf{2 0 1 0}$ & $2,77 \%$ & $7,27 \%$ & $23,32 \%$ & $39,89 \%$ & $26,75 \%$ \\
$\mathbf{2 0 1 1}$ & $1,60 \%$ & $9,40 \%$ & $25,01 \%$ & $39,41 \%$ & $24,58 \%$ \\
$\mathbf{2 0 1 2}$ & $0,61 \%$ & $11,74 \%$ & $21,88 \%$ & $37,95 \%$ & $27,82 \%$ \\
$\mathbf{2 0 1 3}$ & $0,45 \%$ & $4,10 \%$ & $17,29 \%$ & $30,08 \%$ & $48,09 \%$ \\
$\mathbf{2 0 1 4}$ & $3,33 \%$ & $10,73 \%$ & $21,40 \%$ & $35,19 \%$ & $29,35 \%$ \\
$\mathbf{2 0 1 5}$ & $0,41 \%$ & $4,27 \%$ & $16,34 \%$ & $39,95 \%$ & $39,03 \%$ \\
$\mathbf{2 0 1 6}$ & $2,36 \%$ & $10,39 \%$ & $19,91 \%$ & $40,26 \%$ & $27,08 \%$ \\
$\mathbf{2 0 1 7}$ & $0,47 \%$ & $1,11 \%$ & $8,17 \%$ & $43,27 \%$ & $46,98 \%$ \\
$\mathbf{2 0 1 8}$ & $0,54 \%$ & $2,92 \%$ & $13,68 \%$ & $40,23 \%$ & $42,62 \%$ \\
\hline
\end{tabular}

Fonte: Autores (2019).

$\mathrm{Na}$ figura 4, encontra-se os mapas de uso do solo associados com os focos de incêndio para os anos de 2011 e 2015, tendo em vista que esses foram os anos em que sucederam as menores e maiores intensidades de focos. Os mapas foram classificados em vegetação densa, vegetação rala e solo exposto.

Figura 4. Mapas de uso do solo associados aos focos de calor

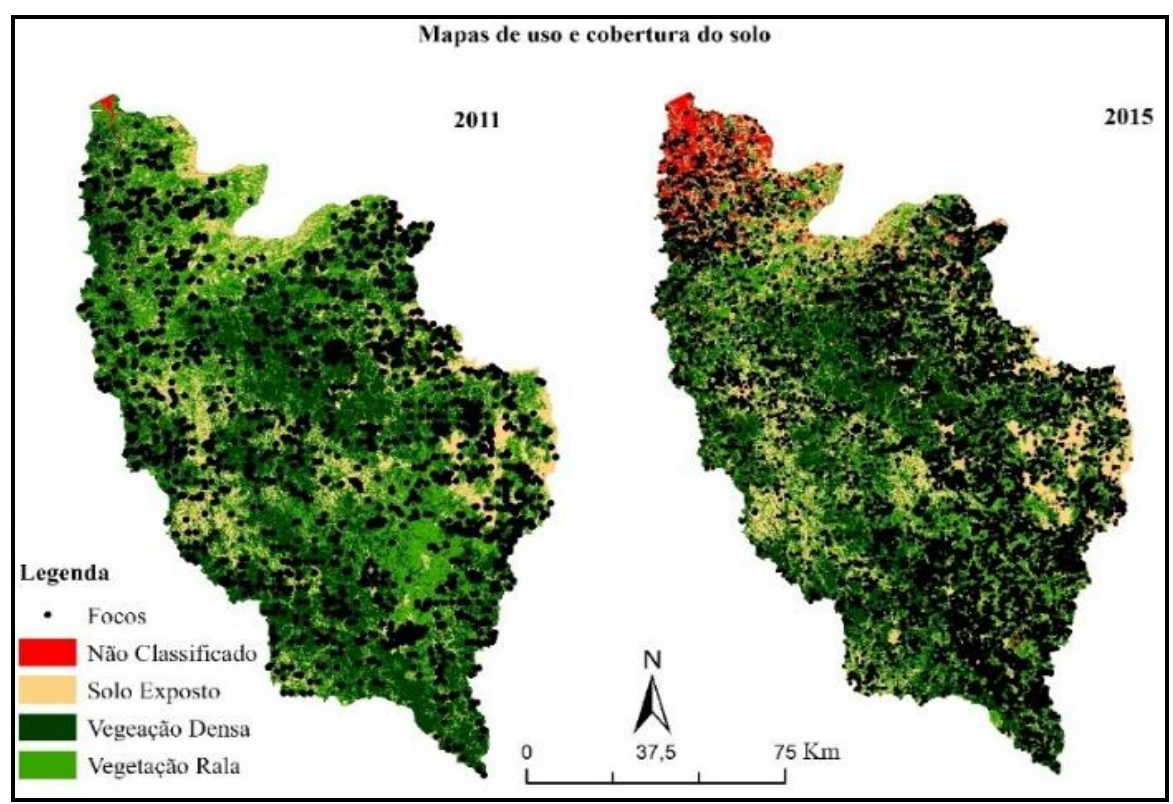

Fonte: Os Autores (2019).

Revista Cerrados, Montes Claros/MG, v. 18, n. 1, p. 373-388, jan./jun.-2020. 
SILVA, R. O.; ARAÚJO, T. C.; SILVA, R. O.; SILVA, C. A. A. C.; OLIVEIRA, A. K. S.; OLIVEIRA, K. A. Sensoriamento remoto na detecção e análise de focos de calor na bacia hidrográfica do Rio Munim

É possível observar que em 2011 os focos de calor estavam associados, principalmente, às áreas de vegetação rala, com um total de 43,13\% dos focos de calor, demostrando a utilização de fogo para o desflorestamento de novas áreas. Esse percentual cai para 34,05\% tratando-se das áreas de solo exposto e 21,82\% para vegetação densa.

No ano de 2015 houve uma mudança no padrão de utilização do fogo, uma vez que nas áreas definidas como vegetação rala decresceram em 25,13\%, passado de 43,13 para $18,00 \%$ do total de focos. Consequentemente, as áreas de solo exposto e de vegetação rala foram acrescidas em 19,58 e 6,55\%, respectivamente.

\section{CONSIDERAÇÕES FINAIS}

Os meses compreendidos entre agosto e novembro possuem uma maior concentração dos graus de perigo Alto e Muito Alto, elevando a possibilidade de ocorrência de incêndios florestais, para a BHRM. Aliado as condições climáticas favoráveis, pois a variação temporal dos focos ocorreu em maior quantidade nos meses associados ao período de estiagem na BHRM.

Os resultados encontrados tendem a contribuir para melhorias e intensificações nas técnicas de prevenção e controle do fogo em áreas de preservação da bacia. Recomenda-se o aprimoramento nas estratégias de combate e fiscalização de queimadas no Maranhão com a finalidade de identificar as causas dos incêndios, principalmente em áreas que apresentaram maiores concentrações de focos de calor.

Além disso, as técnicas de geotecnologias mostraram-se ser eficientes na detecção e análise dos focos de calor, podendo ser uma ferramenta útil para futuros estudos em áreas de difícil acesso e com recorrência de incêndios florestais.

Portanto, de acordo com os resultados obtidos na presente pesquisa indica-se que os estudos futuros na BHRM apresentem temas que busquem identificar as causas da ocorrência das queimadas in loco. Com a abordagem focada na introdução de alguns fatores como: a sócio economia, a análise dos fatores cultural para uso do fogo no Maranhão, em relação as culturas agrícolas, e ainda entre os pequenos e os grandes produtores. Haja vista que as ocorrências de focos foram identificadas em toda as áreas da BHRM, porém, com maiores densidades nas regiões Leste e Sul. 
SIlVA, R. O.; ARAÚJO, T. C.; SIlVA, R. O.; SIlVA, C. A. A. C.; OLIVEIRA, A. K. S.; OLIVEIRA, K. A. Sensoriamento remoto na detecção e análise de focos de calor na bacia hidrográfica do Rio Munim

\section{REFERÊNCIAS}

ABREU, F. A.; SOUZA, J. S. A. Dinâmica Espaço-temporal de Focos de Calor em Duas Terras Indígenas do Estado de Mato Grosso: uma Abordagem Geoespacial sobre a Dinâmica do Uso do Fogo por Xavantes e Bororos. Floresta Ambiente. v.23, n.1, p. 1-10, 2016.

ALVES R. N. B, HOMMA, A. K. O. O fogo na agricultura da Amazonia. Embrapa, Brasília, DF 2020. Disponível em:

https://www.alice.cnptia.embrapa.br/bitstream/doc/1119758/1/LivroRocasemfogop3742.pdf Acesso em: 28 de junho de 2020.

ARAUJO, J. B.; OLIVEIRA, L.C.; VASCONCELOS, S. S.; CORREIA, M. F. Danos Provocados Pelo Fogo Sobre A Vegetação Natural Em Uma Floresta Primária No Estado Do Acre, Amazônia Brasileira. Ciência Florestal, Santa Maria, v. 23, n. 2, p. 297-308, 2013.

BARBOSA, R. I. Distribuição Espacial E Temporal De Focos De Calor Em Roraima Detectados Pelo Noaa-Avhrr (1999-2009). In: Seminário de Atualização em Sensoriamento Remoto e Sistemas de Informações Geográficas Aplicados à Engenharia Florestal, 2010, Curitiba. Anais... Curitiba, PR: 2010, p. 363.

BATISTA, A. C. Detecção de Incêndios Florestais Por Satélites. Revista Floresta, v.34, n.2, p.237-241, 2004.

CABRAL, A. P. S.; Souza W. V. Serviço de Atendimento Móvel de Urgência (SAMU): análise da demanda e sua distribuição espacial em uma cidade do Nordeste brasileiro. Revista Brasileira de Epidemiologia, [S./l.], v. 11, n. 4, p. 530-540, 2008.

DIMYATI, M.; MIZUNO, K.; KITAMURA, T. An analysis of land use/cover change using the combination of MSS Landsat and land use map: a case study in Yogyakarta, Indonesia. Inter. J. Rem. Sen., [S./1.], v. 17, p. 931-944, 1996.

GOMES, C. Geotecnologias da informação e remodelação do espaço urbano regional: Os sistemas de informação geográfica. Interface, [S./l.], v.3, n.3, p. 7-28, 2006.

IBGE - Instituto Brasileiro de Geografia e estatística. Instituto Brasileiro de Geografia e Estatística. Censo Demográfico. 2010. Disponível em: https://cidades.ibge.gov.br/. Acesso em: 20 jul. 2019.

IBGE - Instituto Brasileiro de Geografia e Estatística. Síntese de Informações do Município de Novo Progresso-PA. 2016. Disponível em:

$<$ https://cidades.ibge.gov.br/xtras/temas.php?lang=\&codmun $=150503 \&$ idtema $=16 \&$ search $=\| \mathrm{s}$ $\%$ EDntese-das-informa\%E7\%F5es $>$. Acesso em: 25/ago/2019.

IMESC - Instituto Maranhense de Estudos Socioeconômicos e Cartográficos. Análise da Incidência de Focos de Queimadas nas Terras Indígenas do Estado do Maranhão. In: Relatório Técnico. IMESC. São Luís, 2015. 
SIlVA, R. O.; ARAÚJO, T. C.; SIlVA, R. O.; SIlVA, C. A. A. C.; OLIVEIRA, A. K. S.; OLIVEIRA, K. A. Sensoriamento remoto na detecção e análise de focos de calor na bacia hidrográfica do Rio Munim

IMESC - Instituto Maranhense de Estudos Socioeconômicos e Cartográficos. Relatório: Queimadas maranhenses $-\mathbf{1}^{\circ}$ trimestre 2017. In: Relatório Técnico. IMESC. São Luís, 2017.

INPE - Instituto Nacional de Pesquisas Espaciais. Monitoramento de Queimadas do INPE conta com maior número de satélites. 2007. Disponível em:

<http://www.inpe.br/noticias/noticia.php?Cod_Noticia=1142>. Acesso em: 25/ago/2019.

IPCC [Masson-Delmotte, Valérie et al. (eds.)]. Global warming of $1.5^{\circ} \mathrm{C}-$ Summary for Policymakers. An IPCC Special Report on the impacts of global warming of $1.5^{\circ} \mathrm{C}$ above pre-industrial levels and related global greenhouse gas emission pathways, in the context of strengthening the global response to the threat of climate change, sustainable development, and efforts to eradicate poverty. Working Group I Technical Support Unit, 2018

JUSTINO, F. B. Relação entre "Focos de Calor" e Condições Meteorológicas no Brasil. In: XII Congresso Brasileiro de Meteorologia. Foz de Iguaçu-PR, 2002.

LAZZARINI, G. M. J.; FERREIRA, L. C. C.; FELICÍSSIMO, M. F. G.; OLIVEIRA, L. N.; ALVES, M. V. G. Análise da detecção e ocorrência de queimadas em áreas urbanizadas e entorno. Revista Ciência e Natura, Santa Maria v.38, n.3, 2016.

LEONEL, M. O uso do fogo: o manejo indígena e a piromania da monocultura. Estudos Avançados, [S./1.], v. 14, n. 40, p. 231-250, 2000.

LOPES, A. C.L.; FREITAS, A. V. M.; COSTA, D. O.; BELTRÃO, N. E. S.; TAVARES, P. A.; Análise da distribuição de focos de calor no município de Novo Progresso, Pará. Revista Verde de Agroecologia e Desenvolvimento Sustentável, Pombal, v. 12, n. 2, p. 298-303, 2017.

MARANHÃO. Bacias hidrográficas e climatologia do Maranhão. São Luís, MA: Núcleo Geoambiental, 2016. Disponível em:

https://www.nugeo.uema.br/upnugeo/publicacoes/Bacias\%20Hidrogr\%C3\%A1ficas\%20e\%20 Climatologia\%20-\%20MA. Acesso em: 03 de jul. de 2019.

MARTIN, D. A.; MOODY, J. A. Comparison of soil infiltration rates in burned and unburned mountainous watersheds. Hydrological Processes, [S./l.], v. 15, p. 2893-2903, 2001.

MENESES, P. R.; SANO, E. E. Classificação pixel a pixel de imagens. In: MENESES, P. R.; ALMEIDA, T. (Org). Introdução ao processamento de imagens de sensoriamento remoto. Brasília, DF: Editora da Universidade de Brasília, 2012, p. 198. Disponível em: http://www.cnpq.br/documents/10157/56b578c4-0fd5-4b9f-b82a-e9693e4f69d8. Acesso em: 23 de jul. de 2019.

MIRANDA J. B. Aplicações Do Monitoramento Por Satélite No Controle De Queimadas. In: IV Encontro Nacional sobre Educação Ambiental na Agricultura, 2002, Campinas-SP. Anais... Campinas-SP: EMBRAPA/CNPM, 2002. p. 26. 
SIlVA, R. O.; ARAÚJO, T. C.; SIlVA, R. O.; SIlVA, C. A. A. C.; OLIVEIRA, A. K. S.; OLIVEIRA, K. A. Sensoriamento remoto na detecção e análise de focos de calor na bacia hidrográfica do Rio Munim

NEARY, D. G.; KLOPATEK, C. C.; DEBANO, L. F.; FFOLLIOTT, P. F. Fire effects on belowground sustainability: a review and synthesis. Forest ecology and management, v. 122, n. 1-2, p. 51-71, 1999.

NEPSTAD, D. C.; STICKLER, C. M.; FILHO, B. S.; MERRY, F.; Interações entre o uso da terra, florestas e clima na Amazônia: perspectivas para um ponto de inflexão da floresta no curto prazo. Transações Filosóficas da Royal Society B: Ciências Biológicas, v. 363, n. 1498, p. 1737-1746, 2008.

NOVAIS, J. W. Z.; SANTANNA, F. B.; ARRUDA, P. H. Z.; PEREIRA, O. A.; \& DUTRA, R. Relação entre profundidade óptica de aerossóis e radiação fotossinteticamente ativa e global no cerrado Mato-Grossense. Revista Estudo e Debate, Lajeado, v. 24, n. 1, p. 153-167, 2017.

OLIVEIRA, U. C.; OLIVEIRA, P. S. Mapas de Kernel como Subsídio à Gestão Ambiental: Análise dos Focos de Calor na Bacia Hidrográfica do Rio Acaraú, Ceará, nos Anos 2010 a 2015. Espaço Aberto, v. 7, n.1, p. 87-99, 2017.

PEREIRA, J. A. V.; SILVA, J. B. Detecção de focos de calor no estado da Paraíba: um estudo sobre as queimadas. Revista Geografia Acadêmica, Boa Vista, v.10, n.1, p. 5-16, 2016.

SANTOS, S. R. Q.; DA SILVA, R. B. C.; BARRETO, P. N.; NUNES, H. G. G.; RODRIGUES, R. D. S.; CAMPOS, T. L. D. O. B. Regime térmico e hídrico do solo para área de floresta tropical em anos de El Niño e La Niña, Caxiuanã-PA: estudo de caso. Revista Brasileira de Meteorologia, [S./1.], v. 26, n. 3, p. 367-374, 2011.

SILVA, A. A. C.; VIDAL, J. M. C.; SILVA, R. A.; LACORTE, G. A. Incêndios florestais no Parque Nacional da Serra da Canastra e a implementação de manejo integrado do fogo.

ForScience, v.6, n.2, 2018.

SILVA, M. C.; ROMEU, L. V. L.; FIGUEIREDO, S. M. M.; Análise temporal de focos de calor no Acre: padrões e distribuição. Anais... $7^{\circ}$ Simpósio de Geotecnologias no Pantanal, Jardim, MS, 20 a 24 de outubro 2018. Embrapa Informática. Agropecuária/INPE, p. 154-161.

SILVA, T. B., FRANCA ROCHA, W. J. S., ANGELO, M. F. Quantificação e análise espacial dos focos de calor no Parque Nacional da Chapada Diamantina-BA. Anais XVI Simpósio Brasileiro de Sensoriamento Remoto-SBSR, Foz do Iguaçu, PR, Brasil, v. 13, 2013.

SILVA, V. M. Classificação de imagens por sensoriamento remoto: Análise comparativa das metodologias Pixel a Pixel e Segmentação por Região. 2014. 41f. Monografia (Especialização em Geoprocessamento), Universidade Federal de Minas Gerais, Belo Horizonte, 2014.

SOARES, R. V.; BATISTA, A. C. TETTO, A. F. Incêndios Florestais, Produção Independente. 255p. 2017.

SOUSA, J. S. Áreas de preservação permanente urbanas: mapeamento, diagnósticos, índices de qualidade ambiental e influência no escoamento superficial. Estudo de caso: Revista Cerrados, Montes Claros/MG, v. 18, n. 1, p. 373-388, jan./jun.-2020. 
SIlVA, R. O.; ARAÚJO, T. C.; SIlVA, R. O.; SIlVA, C. A. A. C.; OLIVEIRA, A. K. S.; OLIVEIRA, K. A. Sensoriamento remoto na detecção e análise de focos de calor na bacia hidrográfica do Rio Munim

Bacia do Córrego das Lajes, Uberaba/MG. 2008. 187 f. Dissertação (Mestrado Acadêmico em Engenharia Civil), Universidade Federal de Uberlândia, Uberlândia, 2008.

THOMAZ, E. L.; ANTONELI, V.; DOERR, S. H. Effects of fire on the physicochemical properties of soil in a slash-and-burn agriculture. Catena, v. 122, p. 209-215, 2014.

VETTORAZZI, C. A.; FERRAZ, S. F. B. Uso De Sistema De Informações Geográficas Aplicados À Prevenção E Combate A Incêndios Em Fragmentos Florestais. Série Técnica IPEF, v. 12, n. 32, p. 11-115, 1998.

\section{Autores}

Rennato Oliveira da Silva - Possuiu Graduação em Engenharia Agrícola pela Universidade Federal do Maranhão (UFMA).

Rhuan Oliveira da Silva - Cursa Graduação em Agronomia pela Universidade Federal do Maranhão (UFMA).

Thais de Carvalho Araújo - Cursa Graduação em Ciências Biológicas pela Universidade Federal do Maranhão (UFMA).

Carlos Augusto Alves Cardoso Silva - Possui Graduação em Engenharia Agrícola pela Universidade Federal do Maranhão (UFMA). Atualmente é Mestrando em Engenharia de Sistemas Agrícolas pela Escola Superior de Agricultura "Luiz de Queiroz" (ESALQ/USP).

Ana Karla da Silva Oliveira - Possui Graduação em Engenharia Agrícola pela Universidade Federal do Maranhão (UFMA). Atualmente é Mestranda em Engenharia de Sistemas Agrícolas pela Escola Superior de Agricultura "Luiz de Queiroz" (ESALQ/USP).

Kamilla Andrade Oliveira - Possui Graduação em Engenharia Florestal pela Universidade Federal do Acre (UFAC), Mestrado em Ciências Florestais pela Universidade de Brasília (UnB) e Doutorado em Meteorologia pela Universidade Federal de Viçosa (UFV). Atualmente é Professora da Universidade Federal do Maranhão (UFMA).

Artigo recebido em: 21 de janeiro de 2020.

Artigo aceito em: 26 de junho de 2020.

Artigo publicado em: 30 de junho de 2020. 